\title{
Diagnósticos de enfermería en pacientes con trastorno mental en el Hospital de Día de Malgrat de Mar
}

Nursing Diagnoses in Patients with Mental Disorders Treated in the Day Unit at Hospital Malgrat de Mar

\section{M $^{\mathrm{a}}$ Carmen Moreno-Arroyo ${ }^{1}$, Esther Linares Riera ${ }^{2}$, Guillem Homet Mir ${ }^{3}$, Valenti Agustí Bassa $^{4}$, Natalia Soler Ruiz ${ }^{2}$, Montserrat Puig-Llobet ${ }^{5}$}

\author{
'Departamento de Enfermería Fundamental y Medicoquirúrgica. Escuela de Enfermería, Facultad de Medicina y Ciencias de la Salud de la \\ Universidad de Barcelona. Barcelona, España. \\ ${ }^{2}$ Hospital de Día de Malgrat de Mar. Malgrat de Mar, España. \\ ${ }^{3}$ Clínica de Arenys de Munt. Arenys de Munt, España \\ ${ }^{4}$ Comunidad Terapéutica del Maresme. Barcelona, España. \\ ${ }^{5}$ Departamento de Enfermería de Salud Pública, Salud Mental y Maternoinfantil. Escuela de Enfermería, Facultad de Medicina y Ciencias de la \\ Salud. Universidad de Barcelona. Barcelona, España. \\ Contacto: carmenmoreno@ub.edu \\ Fecha de recepción: 26 de febrero de 2018 / Fecha de aceptación: 13 de mayo de 2018
}

\section{Resumen}

Introducción: Los objetivos planteados fueron: describir el perfil de los usuarios ingresados en el Hospital de Día, identificar los Diagnósticos Enfermeros (DE) más prevalentes a partir de los diagnósticos médicos según el DSM-IVy, analizar la distribución de los DE según los dominios y clases de la taxonomía II de NANDA 2007-2008 en dichos usuarios.

Método: Estudio cuantitativo con un diseño descriptivo retrospectivo. La muestra estuvo configurada por 116 pacientes.

Resultados: Se identificaron un total de 59 DE según la Taxonomía II NANDA, que fueron divididos según diagnósticos médicos psicóticos o neuróticos para identificar la prevalencia de DE en cada grupo. En diagnósticos médicos neuróticos, el DE más destacado fue la ansiedad, seguido de la disposición para mejorar el manejo inefectivo del régimen terapéutico y la baja autoestima. En diagnósticos médicos psicóticos destacó como DE más prevalente la disposición para mejorar el manejo del régimen terapéutico. La mayoría de éstos coincide con los síntomas que describen la patología psiquiátrica.

Conclusiones: El prototipo de persona que cumple las características más comunes entre los usuarios que formaron parte de la muestra, es mujer de media 44 años de edad, soltera, que convive con los padres, pensionista, con diagnóstico de trastorno de personalidad y como patología orgánica asociada.

Palabras clave: diagnóstico de enfermería, dominios y clases taxonomía II NANDA, Hospital de Día, patrones funcionales Gordon, historia de enfermería.

\begin{abstract}
The proposed objectives were: to describe the profile of the users admitted to the Day Unit, to identify the most prevalent Nursing Diagnoses (ND) based on the medical diagnoses according to the DSM-IV and analyze the distribution of the ND according to the domains and classes of the NANDA taxonomy II 2007-2008 in said users. It is a quantitative study with a retrospective descriptive design. The sample was configured by 116 patients. The data was obtained during the period from January to September 2016. A total of 59 ND were identified according to NANDA Taxonomy II, which were divided according to psychotic or neurotic medical diagnoses to identify the prevalence of ND in each group. In neurotic medical diagnoses, the most prominent ND was anxiety, followed by the willingness to improve the ineffective management of the therapeutic regimen and low self-esteem. In psychotic medical diagnoses, the disposition to improve the management of the therapeutic regimen was highlighted as the most prevalent ND, followed by the disturbance of the thought process and the sensory perception disorder together with the ineffective maintenance of health. Most of these match the symptoms that depict the psychiatric pathology.
\end{abstract}

Keywords: nursing diagnoses, domains and classes taxonomy II NANDA, day unit, functional patterns, nursing history. 


\section{Introducción}

Los trastornos mentales han sido definidos mediante una gran variedad de conceptos, siendo cada uno un indicador útil para un tipo de trastorno, pero ninguno equivalente a cada concepto y caso, requiriendo una definición distinta para cada trastorno ${ }^{1}$. Declaraciones recientes de la $\mathrm{OMS}^{2}$ exponen que el $25 \%$ de la población sufre un trastorno mental a lo largo de su vida, previendo que aumentaran las cifras de forma considerable en los próximos años. Estos representan el 12,5\% de todas las patologías, siendo este porcentaje superior al del cáncer y los trastornos cardiovasculares. Según un análisis de la Agència de Qualitat i Avaluació Sanitàries de Catalunya ${ }^{3}$, no existen datos recogidos sobre el porcentaje de personas atendidas en hospitales de día, pero sí en centros de salud mental de adultos (CSMA) y unidades de agudos y subagudos. Los datos indican que los servicios de atención ambulatoria de adultos cuentan con 73 CSMA y una población de referencia de 6109571 personas $^{3}$. De estas, un 2,77\% fue atendido en algún CSMA a lo largo del año 2015, lo que supone un total de más de 169000 personas $^{3}$. En relación a la hospitalización de agudos, se realizaron 22739 hospitalizaciones, un $86,4 \%$ en la Unidad de Agudos y un 13,6\% en la Unidad de Subagudos, con un crecimiento del $15,8 \%$ respecto al año $2014^{3}$. Los hospitales de día son una adquisición reciente de la psiquiatría (siglo $\mathrm{XX}$ ) que se engloba dentro de los avances más importantes de este ámbito. La aparición de dichas estructuras terapéuticas permitió la reintegración social de los usuarios, gracias a la transformación de las instituciones y la aparición de nuevas modalidades terapéuticas encaminadas hacia la salud mental comunitaria $^{4,5}$. El primer hospital de día fue en la Unión Soviética en la década de los $30 \mathrm{y}$, casi de forma simultánea, se establecieron también en Inglaterra y Norteamérica ${ }^{4}$. En este contexto, los profesionales enfermeros tienen un papel relevante en potenciar el vínculo terapéutico, mejorar la adherencia al tratamiento, trabajar la consciencia de enfermedad, potenciar la participación de las familias y trabajar la promoción, prevención y educación para la salud ${ }^{5}$. En esta línea, y para facilitar la planificación de los cuidados de enfermería, surge en 1999 como consenso del Grupo de Trabajo de Salud Mental y Comunitaria de Cataluña, un registro para realizar una valoración enfermera basado en los patrones funcionales de Marjory Gordon $^{6}$, a partir de los cuales se modificó la estructura de la taxonomía North American Nursing Diagnosis Association $^{7}$ (NANDA). Esta estructura ha sido muy utilizada en los procesos de atención de enfermería; no obstante, son escasos los estudios realizados. De ello, se derivan los objetivos de este estudio: describir el perfil de los usuarios del Hospital de Día de Malgrat de Mar, identificar los diagnósticos enfermeros (DE) más prevalentes a partir de los diagnósticos médicos según el DSM-IV y analizar la distribución de los DE según los dominios y clases de la taxonomía II de NANDA $^{7}$ 2007-2008 en dichos usuarios.

\section{Material y métodos}

\section{Diseño}

Estudio cuantitativo con un diseño descriptivo retrospectivo realizado en el Hospital de Día de Malgrat de Mar, que forma parte del consorcio sanitario de Salud Mental del Maresme (Comunitat Terapèutica del Maresme) y que ofrece servicio asistencial a una población de 2200 habitantes. Tiene una capacidad de 20 plazas con una rotación de 12 ingresos y 12 altas mensuales.

\section{Población y muestra}

Los sujetos que configuraron la muestra fueron los usuarios ingresados en el Hospital de Día de Malgrat de Mar en el periodo de enero a septiembre del 2016. El tamaño de la muestra fue de 116 usuarios. Los criterios de inclusión fueron: usuarios ingresados en el Hospital de Día, mayores de 18 años y diagnóstico de salud mental con seguimiento desde el Centro de Salud Mental de Adultos (CSMA). Los criterios de exclusión fueron: usuarios que presentaron agudización del trastorno mental durante el ingreso.

\section{Variables y recogida de datos}

Se elaboró una plantilla de registro informatizada con las siguientes variables: edad, sexo, diagnóstico psiquiátrico, patologías asociadas, estado civil, pensión y diagnóstico de enfermería según la taxonomía II NANDA ${ }^{7}$. Se utilizó dicha taxonomía, ya que, a pesar de existir una actualización posterior, las autoras estaban más familiarizadas con dicha taxonomía. Los datos se obtuvieron de la revisión de las historias clínicas y se analizaron mediante el programa SPSS 16.0. Se realizaron análisis descriptivos de frecuencias. Se solicitaron los permisos pertinentes a la dirección médica del Hospital de Día de la Comunitat Terapèutica del Maresme, así como también se pasó el Comité de Ética e Investigación del centro. 


\section{Resultados}

La media de edad entre los usuarios que formaron parte del estudio fue de 44 años, un 52,58\% eran mujeres y un $47,41 \%$, hombres. En referencia al estado civil, un $47 \%$ de los usuarios eran solteros, un $26,72 \%$, separados, un $24,14 \%$, casados y el $1,72 \%$ restante, viudos. En cuanto al lugar de residencia, un 19,83\% convivía con los padres, un $15,52 \%$ vivían solos y un $14,66 \%$, con la madre, los usuarios restantes convivían en diferentes estructuras familiares menos destacables. En relación a las patologías orgánicas asociadas, observamos que el 25,53\% presentaba dislipemia, seguido de un $14,89 \%$ con hipertensión arterial y un 10,64\% con ambas patologías conjuntas. En base a su estado económico, se detalla en la tabla 1. En relación al trastorno psiquiátrico según el DSM-IV, el diagnóstico médico más frecuente fueron los trastornos de personalidad seguidos de la esquizofrenia paranoide (tabla 2). El total de diagnósticos médicos fueron divididos en psicóticos y neuróticos. En relación a los diagnósticos médicos neuróticos, el DE más destacado fue la ansiedad con un 78,57\%, y en los diagnósticos médicos psicóticos, el DE más prevalente fue la disposición para mejorar el manejo del régimen terapéutico con un $82,2 \%$. En la tabla 3 se clasifican los diagnósticos por dominios y clases según la taxonomía II $\mathrm{NANDA}^{7}$ 2007-2008, destacando las frecuencias y porcentajes totales. Si partimos del $50 \%$ como punto de corte, observamos que los 6 diagnósticos más prevalentes son la ansiedad, el manejo inefectivo del régimen terapéutico, el mantenimiento inefectivo de la salud, la baja autoestima, el déficit de actividades recreativas y el afrontamiento inefectivo. Los dominios donde hay un número mayor de DE son el dominio 9: Afrontamiento y tolerancia al estrés (n:11) 18,64\%; el dominio 4: Actividad/reposo (n:10) 16,64\% y; el dominio 5: Percepción/cognición (n:6) 10,16\%.

\section{Discusión}

Se identificaron 59 DE de los 188 diagnósticos que recoge la taxonomía II NANDA 2007-2008. Se observó un reducido número de diagnósticos prevalentes, siendo el principal la ansiedad con un $93,97 \%$, el manejo inefectivo del régimen terapéutico con un 70,69\% y el mantenimiento inefectivo de la salud con un $68,10 \%$. Destacar que los resultados obtenidos se vieron condicionados por el elevado número de usuarios con diagnóstico médico neurótico, motivo por el cual destacan las características principales y la sintomatología de la neurosis. No se hallaron estudios publicados y desarrollados en un hospital de día bajo la misma perspectiva. Sin embargo, encontramos un estudio realizado en el CSMA de Badalona y Santa Coloma de Gramanet $^{8}$, que también analiza los DE, aunque en pacientes con trastorno mental severo (TMS). En dicho estudio, se obtuvo como resultado que los diagnósticos más prevalentes eran el desequilibrio nutricional por exceso, el déficit de autocuidado en el baño e higiene y el déficit de actividades recreativas. Si lo comparamos con nuestro estudio, observamos que el único DE que coincide es el déficit de actividades recreativas, siendo este unos de los diagnósticos de enfermería con más incidencia en ambos. Las características que definen este diagnóstico van muy ligadas a cualquier patología mental, al experimentar una disminución de la estimulación, del interés o de la participación en actividades recreativas o de ocio, relacionando todo ello con la apatía, la anhedonia y la falta de motivación que presentan la mayoría de usuarios. En relación al perfil de los usuarios ingresados en el hospital de día y analizando los datos obtenidos, observamos también que la diferencia entre hombres y mujeres es mínima, de un 5,17\%, siendo superior en el sexo femenino. Si lo comparamos con el estudio realizado en el CSMA Badalona y Santa Coloma de Gramanet ${ }^{8}$, observamos que los datos no coinciden, ya que en dicho caso la diferencia entre sexos es de un $36,4 \%$, siendo superior el masculino. Sucede lo mismo con la media de edad de nuestra muestra, 44 años, frente a los 39,8 del estudio mencionado. Pero en referencia a la patología más prevalente, coinciden ambos en determinar que la esquizofrenia paranoide es el trastorno más frecuente. Se encontró otro estudio que coincide con nuestros resultados ${ }^{9}$, ya que la esquizofrenia fue el diagnóstico médico más prevalente así como el sexo femenino de los pacientes ingresados en la unidad; en este último caso, debido a un conjunto de factores sociales y culturales. En base al estado económico de los usuarios de nuestro estudio, la gran mayoría son pensionistas, pero es importante destacar que los usuarios con diagnóstico médico neurótico (pendientes de la evaluación del ICAM para la obtención de una pensión económica) sobrepasan en un 14,71\% a los usuarios con diagnóstico médico psicótico. Frente a estos datos, no hemos encontrado estudios comparativos en los cuales se haya estudiado esta variable con este tipo de usuarios, pero existe un estudio de costes y carga de la depresión en Cataluña ${ }^{10}$, del año 2006, en el cual se ha estimado que de las 161800 personas con incapacidad permanente valorada por el ICAM en Cataluña, en el año 2007, 17302 (un 10,69\%) están diagnosticadas de depresión (diagnóstico médico 
neurótico). En las revisiones del ICAM, 2 de cada 3 propuestas de incapacidad permanente de personas con diagnóstico de depresión fueron aceptadas. En cuanto a la distribución de los DE por dominios y clases, destacar que los dominios con un mayor número de diagnósticos son el dominio 9: Afrontamiento y tolerancia al estrés; el dominio 4: Actividad/reposo y el dominio 5: Percepción/cognición. No se da ningún DE en el dominio 13: Crecimiento, al no presentar importancia en estos usuarios. Si comparamos nuestro estudio con el hecho en los CSMA de Badalona y Santa Coloma de Gramanet ${ }^{8}$, obtenemos resultados similares en relación a los dominios con más prevalencia, ya que en dicho estudio el número más elevado de diagnósticos se encuentra en los dominios 4, 9 y 7, y no se da ninguno en el dominio 13 . No pasa lo mismo respeto a la prevalencia de los DE en ambos estudios. En el nuestro, del punto de corte de $30-50 \%$, solo encontramos 7 DE y en $<30 \%$, los 46 DE restantes, lo cual refleja que existe una alto porcentaje y frecuencia de DE prevalentes en los usuarios ingresados en el hospital de día, hecho contrario al estudio realizado en el CSMA de Badalona y Santa Coloma de Gramanet ${ }^{8}$, donde entre el punto de corte de $20-49 \%$ solo encuentran 10 diagnósticos, entre el 10-19\%, 14 y en $<10 \%$ los 15 restantes, lo cual refleja la no obtención de un gran porcentaje y frecuencia de DE prevalentes en los usuarios con TMS estudiados en dicho estudio. Probablemente, esto se deba a las distintas características de los usuarios y a la presentación de su clínica psiquiátrica, más agudizada en el hospital de día que en los CSMA, donde la sintomatología no está en fase tan aguda y no hay DE que despunten frente a otros, al estar clínicamente más estables.

\section{Conclusiones}

Los usuarios más comunes fueron de una mujer de 44 años de edad, soltera, que convive con los padres, pensionista, con diagnóstico de trastorno de personalidad, y como patología orgánica asociada, dislipemia. Se destaca la elevada ansiedad y la falta de herramientas para gestionar el manejo de la enfermedad, independientemente que esté diagnosticado de una patología mental neurótica o psicótica. En el perfil del usuario con patología psiquiátrica, queda afectada el área emocional, por ello, los DE más prevalentes de este estudio coinciden con los que mejor describen los síntomas de la patología psiquiátrica. En base a la distribución de los DE según los dominios y clases, concluimos con la identificación de $59 \mathrm{DE}$ de los 188 totales de la taxonomía II NANDA. Los dominios que destacan por un mayor número de $\mathrm{DE}$ son el dominio 9: Afrontamiento y Tolerancia al estrés, el dominio 4: Actividad/Reposo y; el dominio 5: Percepción/Cognición.

\section{Agradecimientos}

A la Comunitat Terapèutica del Maresme y a todo el equipo del Hospital de Día de Malgrat de Mar. A David Bouzo y Abel Salon, por la ayuda mostrada a nivel estadístico. A las personas atendidas que nos han permitido llevar a cabo este proyecto.

\section{Bibliografía}

1. American Psychiatric Association (APA). Manual Diagnóstico y Estadístico de los Trastornos Mentales DSM-IV-TR. Barcelona: Masson; 2002.

2. Organización Mundial de la Salud (OMS). Trastornos mentales, 2017.Disponible en:

http://www.who.int/mediacentre/factsheets/fs396/es/. Consultado el 2 de mayo de 2017

3. Observatori del Sistema de Salut de Catalunya. Central de resultats. Àmbit de Salut Mental. Dades 2015. Barcelona: Agència de Qualitat i Avaluació Sanitàries de Catalunya. Depertament de Salut. Generalitat de Catalunya; 2016.

4. Sepúlveda R. Hospital de Día en psiquiatría. Evolución de la experiencia mundial y estado de la situación en Chile. [Internet] Monografías de gestión en psiquiatría y salud mental. Universidad de Santiago de Chile; 2001. Disponible en:

http://www.bvsde.paho.org/texcom/cd050644/naranjo.pdf. Consultado el 2 de mayo de 2017.

5. INSALUD. Guía de gestión del hospital de día psiquiátrico. Madrid:INSALUD; 1999.

6. Gordon M. Diagnóstico enfermero. Proceso y aplicación. $3^{\mathrm{a}}$ ed. Madrid: Mosby, Doyma; 1996.

7. Nanda International. Diagnósticos enfermeros. Definiciones y clasificación 2007-2008. $2^{\text {a }}$ ed. Barcelona. Elsevier; 2007

8. Lluch MT, Sabadell M, Puig M, Cencerrado $M^{a} A$, Navas D, Ferret $M$, Moreno T, Ugalde M. Diagnósticos enfermeros en pacientes con trastorno mental severo con seguimiento de enfermería en centros de salud mental. Rev. Presencia 2009 jul-dic, 5(10). Disponible en:

http://www.index-f.com/presencia/n10/p7151r.php. Consultado el 4 de octubre de 2017.

9. Vicente I, Fombellida CI, Fernández LC, Sandoval O. Estudio de la calidad asistencial en el hospital de día psiquiátrico de Salamanca. Rev. enferm. CyL.2010; 2(1). Disponible en: file:///C:/Users/Usuari/Downloads/37-130-1-PB\%20(3).pdf. Consultado el 10 de setembre de 2017.

10. Estudio de costes y carga de la depresión en Cataluña (CostDep2006). Generalitat de Cataluña. Departament de Salut. 2011. Disponible en: https://scientiasalut.gencat.cat/bitstream/handle/11351/2670 / estudio_costes _ carga _ depresion _ cat alu\%C3\%B1a_2011_cas.pdf?sequence=6- Consultado el 4 de octubre de 2017. 
Tabla 1: Clasificación de los usuarios totales ingresados en Hospital de Día, según su estado económico

\begin{tabular}{cccccc}
\hline Neuróticos & $\mathbf{N = 7 0}$ & $\mathbf{\%}$ & Psicóticos & $\mathbf{N = 4 6}$ & $\mathbf{\%}$ \\
\hline Pensionista & 44 & 62,85 & Pensionista & 39 & 84,78 \\
ICAM & 20 & 25,57 & ICAM & 5 & 10,86 \\
Baja Laboral & 6 & 8,5 & Baja Laboral & 2 & 4,34 \\
\hline
\end{tabular}

ICAM: (Institut Català d'Avaluació Mèdica del Departament de Salut, GENCAT

Tabla 2: Diagnósticos médicos de los usuarios ingresados en el Hospital de Día de Malgrat

$\begin{array}{lll}\text { Diagnósticos médicos } & \text { N } & \%\end{array}$

Trastorno Límite de Personalidad 20

Trastorno Depresivo

Trastorno Bipolar

Trastorno Adaptativo 3

Trastorno de Somatización/Conversivo

1

0,86

Sintomatología Ansiosa

Trastorno histriónico de la Personalidad 3

Trastorno de la Personalidad Cluster B + C

Trastorno Obsesivo Compulsivo (TOC)

Trastorno de la Personalidad Cluster B

Trastorno Esquizoafectivo

Esquizofrenia Paranoide

Trastorno Psicótico no Especificado +TOC

Trastorno Psicótico

Trastorno de la Personalidad Esquizoide

+ TOC + Trastorno Depresivo

Esquizofrenia subcrónica

$1 \quad 0,86$

Psicosis Inespecificada

1

0,86

Total

116

100 
Tabla 3: Diagnósticos de enfermería, distribución por dominios y clases según la taxonomía Il de NANDA 2007-2008

\begin{tabular}{|c|c|c|c|}
\hline Diagnósticos enfermeros & $\mathbf{N}(\%)$ & Dominio & Clase \\
\hline Manejo inefectivo del régimen terapéutico 00078 & $82(70,69)$ & 1 & 2 \\
\hline Mantenimiento inefectivo de la salud 00099 & $79(68,10)$ & 1 & 2 \\
\hline Incumplimiento del tratamiento 00079 & $23(19,83)$ & 10 & 3 \\
\hline Disposición para mejorar el manejo del régimen terapéutico 00162 & $99(85,34)$ & 1 & 2 \\
\hline Tendencia a adoptar conductas de riesgo para la salud 00188 & $42(36,21)$ & 9 & 2 \\
\hline Desequilibrio nutricional por defecto 00002 & $12(10,34)$ & 2 & 1 \\
\hline Desequilibrio nutricional por exceso 00001 & $32(27,59)$ & 2 & 2 \\
\hline Disposición para mejorar la nutrición 00163 & $28(24,14)$ & 2 & 1 \\
\hline Riesgo de desequilibrio nutricional por exceso 00003 & $19(16,38)$ & 2 & 1 \\
\hline Déficit de autocuidado: Alimentación 00102 & $28(24,14)$ & 4 & 5 \\
\hline Deterioro de la eliminación urinaria 00016 & $4(3,45)$ & 3 & 1 \\
\hline Diarrea 00013 & $3(2,59)$ & 3 & 2 \\
\hline Estreñimiento 00011 & $8(6,90)$ & 3 & 2 \\
\hline Riesgo de estreñimiento 00015 & $31(26,72)$ & 3 & 2 \\
\hline Estreñimiento subjetivo 00012 & $0(0,00)$ & 3 & 2 \\
\hline Déficit de actividades recreativas 00097 & $67(57,76)$ & 4 & 2 \\
\hline Sedentarismo 00168 & $43(37,07)$ & 4 & 2 \\
\hline Déficit autocuidado: Vestido/acicalamiento 00109 & $14(12,07)$ & 4 & 5 \\
\hline Déficit de autocuidado: Baño/higiene 00108 & $18(15,52)$ & 4 & 5 \\
\hline Intolerancia a la actividad 00093 & $14(12,07)$ & 4 & 4 \\
\hline Fatiga 00093 & $6(5,17)$ & 4 & 3 \\
\hline Deprivación de sueño 00096 & $12(10,34)$ & 4 & 1 \\
\hline Insomnio 00095 & $54(46,55)$ & 4 & 1 \\
\hline Disposición para mejorar el sueño 00165 & $56(48,28)$ & 4 & 1 \\
\hline Trastorno de los procesos de pensamiento 00130 & $48(41,38)$ & 5 & 4 \\
\hline Confusión crónica 00129 & $1(0,86)$ & 5 & 4 \\
\hline Deterioro de la comunicación verbal 00051 & $15(12,93)$ & 5 & 5 \\
\hline Trastorno de la percepción sensorial 00122 & $29(25,00)$ & 5 & 3 \\
\hline Confusión aguda 00128 & $2(1,72)$ & 5 & 4 \\
\hline
\end{tabular}


Conocimientos deficientes 00126

Desesperanza 00124

Riesgo de suicidio 00150

Riesgo de violencia autodirigida 00140

Baja autoestima crónica 00119

Trastorno de la imagen corporal 00118

Trastorno de la identidad personal 00121

Desempeño inefectivo del rol 00055

Aislamiento social 00053

Cansancio rol de cuidador 00061

Riesgo de violencia dirigida a otros 00138

Riesgo de cansancio rol de cuidador 00062

Riesgo de soledad 00054

Interrupción de los procesos familiares 00060

Disfunción sexual 00059

Patrón sexual inefectivo 00065

Temor 000149

Ansiedad 00146

Afrontamiento inefectivo 00069

Duelo 00136

Afrontamiento familiar incapacitante 00073

Afrontamiento familiar comprometido 00074

Negación inefectiva 00072

Síndrome postraumático 00141

Riesgo de síndrome postraumático 00145

Disposición parar mejorar el afrontamiento 00158

Automutilación 00151

Riesgo de automutilación 00139

Sufrimiento espiritual 00066

Riesgo de sufrimiento espiritual 00067

\begin{tabular}{|c|c|}
\hline $3(2,59)$ & 5 \\
\hline $26(22,41)$ & 6 \\
\hline $12(10,34)$ & 11 \\
\hline $10(8,62)$ & 11 \\
\hline $76(65,52)$ & 6 \\
\hline $6(5,17)$ & 6 \\
\hline $0(0,00)$ & 6 \\
\hline $33(28,45)$ & 7 \\
\hline $49(42,24)$ & 12 \\
\hline $18(15,52)$ & 7 \\
\hline $7(6,03)$ & 11 \\
\hline $23(19,83)$ & 7 \\
\hline $33(28,45)$ & 6 \\
\hline $19(16,38)$ & 7 \\
\hline $2(1,72)$ & 8 \\
\hline $7(6,03)$ & 8 \\
\hline $31(26,72)$ & 9 \\
\hline $109(93,97)$ & 9 \\
\hline $59(50,86)$ & 9 \\
\hline $10(8,62)$ & 9 \\
\hline $21(18,10)$ & 9 \\
\hline $53(45,69)$ & 9 \\
\hline $21(18,10)$ & 9 \\
\hline $0(0,00)$ & 9 \\
\hline $1(0,86)$ & 9 \\
\hline $26(22,41)$ & 9 \\
\hline $9(7,76)$ & 11 \\
\hline $28(24,14)$ & 11 \\
\hline $0(0,00)$ & 10 \\
\hline $0(0,00)$ & 10 \\
\hline
\end{tabular}

1

3

3

2

3

3

3

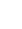

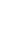

1

2

2

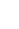

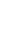

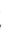

2

